https://doi.org/10.15407/ujpe66.3.206

M. MOLLA GESSESSE

Department of Physics, Aksum University

(P. O. Box 1010, Axum, Ethiopia; e-mail: mokemolla@gmail.com)

\title{
ELECTRICALLY PUMPED TWO-MODE LASER DYNAMICS
}

\begin{abstract}
The statistical and squeezing properties of the cavity light produced by a three-level laser are studied. In the laser, $N$ three-level atoms in an open cavity are coupled with a two-mode vacuum reservoir and are pumped to the top level by means of the electron bombardment. Applying the steady-state solutions of the equations of evolution of the expectation values of the atomic operators and the quantum Langevin equations for the cavity mode operators, we have obtained the global and local photon statistics for single-mode cavity light beams and for two-mode cavity light. It is found that the global mean photon number and the global photon-number variance of the light emitted from the top are greater than those for the light emitted from intermediate level. The cavity lights emitted from the top and intermediate levels can be separately in a chaotic state under certain conditions. However, the two-mode cavity light is in a squeezed state under certain conditions. We have established that the maximum quadrature squeezing of the two-mode cavity light to be about $46 \%$ below the coherent-state level. The presence of the vacuum reservoir noise has the effect of increasing the photon-number variance and decreasing the quadrature squeezing of the cavity light but has no effect on the mean photon number. We have shown that the local mean photon number and photon number variance of the cavity light approach the global mean photon number and photon number variance of the cavity light as the frequency interval increases.
\end{abstract}

Ke ywords: stimulated emission decay constant, photon statistics, power spectrum, photon number fluctuations, quadrature squeezing.

\section{Introduction}

In recent years, a three-level cascade laser has attracted a great deal of interest in connection with its potential as a source of a squeezed light [1-4], which is expected due to the correlation between the two photons generated, when an atom decays from the top to the bottom level via the intermediate level [5]. A three-level laser may be defined as a quantum optical system in which three-level atoms in a cascade configuration, initially prepared in a coherent superposition of the top and bottom levels, are injected into a cavity coupled to a vacuum reservoir via a singleport mirror. A three-level laser may have additional or modified features. One interesting additional feature of a three- level laser involves the coupling of the top and bottom levels of the atoms injected into the cavity by a strong coherent light. When a three-level atom in a cascade configuration makes a transition

(C) M. MOLLA GESSESSE, 2021 from the top to the bottom level via the intermediate level, two photons are generated. If the two photons have the same frequency, then the three-level atom is called degenerate three-level atom otherwise it is called nondegenerate.

The statistical and the squeezing properties of the light generated by a three-level laser have been investigated by several authors [2-9]. Some authors have studied a three-level laser in which the top and ground levels of the atoms injected into the cavity are coupled by strong coherent light $[10,11]$. Three-level lasers in which the crucial role is played by the coherent superposition of the top and bottom levels of the injected atoms have been studied in $[7,11]$. These studies showed that this quantum optical system can generate light in a squeezed state under certain conditions.

Preparing the three-level atoms in a coherent superposition of the top and bottom levels before they are injected into a laser cavity is not a simple task. Moreover, it may not possible to know whether

ISSN 2071-0194. Ukr. J. Phys. 2021. Vol. 66, No. 3 
the atoms have decayed spontaneously to levels other than the intermediate or bottom level before they are removed from the laser cavity $[3,6]$. In addition, when the top and bottom levels of the three-level lasers are coupled by strong coherent light, the atoms may decay from the top to bottom levels. This decay may decrease the mean photon number of the cavity light.

Fesseha and Solomon $[12,13]$ have studied a threelevel laser in which three-level atoms available in a closed cavity are pumped from the bottom to the top level by the electron bombardment. They carried out their analysis by putting the vacuum noise operators in the normal order which renders the vacuum reservoir to be a noiseless physical entity. They have found that the light generated by the three-level laser operating far below the threshold is in a squeezed state, with the maximum quadrature squeezing being $50 \%$ below the vacuum-state level. In addition, they have shown that the quadrature squeezing of the output light is equal to that of the cavity light. On the other hand, their study showed that the local quadrature squeezing is greater than the global quadrature squeezing.

Furthermore, Fesseha [3] has studied a three-level laser in which three-level atoms available in a closed cavity are pumped from the bottom to the top level by coherent light. He carried out his analysis by putting the vacuum noise operators in the normal order. He has found that the light generated by the three-level laser operating under certain conditions is in a squeezed state, with the maximum quadrature squeezing being about $43 \%$ below the vacuum-state level. In addition, he has shown that the quadrature squeezing of the output light is equal to that of the cavity light. On the other hand, this study shows that the local quadrature squeezing is greater than the global quadrature squeezing.

Recently, Mekonnen [6] has also studied a threelevel laser in which three-level atoms available in a closed cavity are pumped from the bottom to the top level by coherent light. He carried out his analysis by putting the vacuum noise operators in an arbitrary order. He has found that the light generated by the three-level laser operating under certain conditions is in a squeezed state, with the maximum quadrature squeezing being less than $43 \%$ below the vacuumstate level. In addition, he has also shown that the effect of the vacuum reservoir noise is to increase the photon number variance and to decrease the quadra- ture squeezing. However, the vacuum reservoir noise has no any effect on the mean photon number.

This paper essentially has two parts. In the first part, we will study the statistical properties of the light generated by three-level atoms available in an open cavity and pumped to the top level by the electron bombardment. We will carry out our calculation by taking the noise operators associated with the vacuum reservoir in an arbitrary order. We first determine the quantum Langevin equations and the equations of evolution of the atomic operators. Moreover, we determine the solutions of the equations of evolution of the atomic operators and the quantum Langevin equations for the cavity mode operators. Then, applying the resulting solutions, we calculate the photon statistics of the single-mode and two-mode cavity lights.

In the second part, we will analyze the squeezing properties of the two-mode cavity light produced by three-level lasers in which the three-level atoms available in an open cavity are pumped to the top level from the bottom and ground level by the electron bombardment.

\section{Equations of Evolution}

We consider $N$ three-level atoms available in a cavity coupled to a two-mode vacuum reservoir via a single port mirror. We study the case where the cavity light and the atoms are interacting with a twomode vacuum reservoir. We represent the top, intermediate, bottom, and ground levels of the $k^{\text {th }}$ threelevel atom in the cascade configuration by $\left|a_{k}\right\rangle,\left|b_{k}\right\rangle$, $\left|c_{k}\right\rangle$, and $\left|d_{k}\right\rangle$, respectively. We assume the transitions $\left|a_{k}\right\rangle \rightarrow\left|b_{k}\right\rangle$ and $\left|b_{k}\right\rangle \rightarrow\left|c_{k}\right\rangle$ to be dipole allowed, with direct transitions $\left|a_{k}\right\rangle \rightarrow\left|c_{k}\right\rangle$ to be dipole forbidden. In addition, we consider the case where the cavity modes are at resonance with the two transitions $\left|a_{k}\right\rangle \rightarrow\left|b_{k}\right\rangle$ and $\left|b_{k}\right\rangle \rightarrow\left|c_{k}\right\rangle$. We also consider the case where the atoms are pumped from the ground level $\left|d_{k}\right\rangle$ and bottom level $\left|c_{k}\right\rangle$ to the top level $\left|a_{k}\right\rangle$ at the rates of $r_{d a}$ and $r_{c a}$, respectively, by means of the electron bombardment. A three-level atom may make a transition from the level $\left|a_{k}\right\rangle$ to $\left|b_{k}\right\rangle$ and from $\left|b_{k}\right\rangle$ to $\left|c_{k}\right\rangle$, by emitting photon of the same frequency $\omega$. To this end, we prefer to call the light emitted by the $N$ three-level atoms from the top energy level light mode $a$ and the light emitted from the intermediate energy level light mode $b$. In addition, the atom may 
decay from levels $\left|a_{k}\right\rangle,\left|b_{k}\right\rangle$, and $\left|c_{k}\right\rangle$ spontaneously to the ground level $\left|d_{k}\right\rangle$ (assumed to be the same for all transitions) at the rate of, say, $\gamma[14,15]$. In the rotating wave and electric dipole approximations, the interaction of one of the $k^{\text {th }}$ atoms to the cavity light in the rotating frame of reference is described by the Hamiltonian $[2,4,6]$

$\hat{H}=i g\left[\left|a_{k}\right\rangle\left\langle b_{k}\left|\hat{a}-\hat{a}^{\dagger}\right| b_{k}\right\rangle\left\langle a_{k}|+| b_{k}\right\rangle\left\langle c_{k}\left|\hat{b}-b^{\dagger}\right| c_{k}\right\rangle\left\langle b_{k}\right|\right]$,

where $g$, which is assumed to be the same for both transitions, is a coupling constant between the atom and the cavity mode, $\hat{a}$ is the annihilation operator for the cavity mode, and $\left|b_{k}\right\rangle\left\langle a_{k}\right|\left(\left|c_{k}\right\rangle\left\langle b_{k}\right|\right)$ is the atomic lowering operator. The master equation describing the interaction of three-level atom and cavity modes with one another andwith the two-mode vacuum reservoir is found to be [2]

$\frac{d \hat{\rho}}{d t}=-i[\hat{H}, \hat{\rho}]+\frac{\kappa}{2}\left[2 \hat{a} \hat{\rho} \hat{a}^{\dagger}-\hat{a}^{\dagger} \hat{a} \hat{\rho}-\hat{\rho} \hat{a}^{\dagger} \hat{a}+2 \hat{b} \hat{\rho} \hat{b}^{\dagger}-\hat{b}^{\dagger} \hat{b} \hat{\rho}-\right.$

$\left.-\hat{\rho} \hat{b}^{\dagger} \hat{b}\right]+\frac{\gamma}{2}\left[2\left|d_{k}\right\rangle\left\langle a_{k}|\hat{\rho}| a_{k}\right\rangle\left\langle d_{k}|-| a_{k}\right\rangle\left\langle a_{k}\right| \hat{\rho}-\right.$

$-\hat{\rho}\left|a_{k}\right\rangle\left\langle a_{k}|+2| d_{k}\right\rangle\left\langle b_{k}|\hat{\rho}| b_{k}\right\rangle\left\langle d_{k}|-| b_{k}\right\rangle\left\langle b_{k}\right| \hat{\rho}-$

$\left.-\hat{\rho}\left|b_{k}\right\rangle\left\langle b_{k}|+2| d_{k}\right\rangle\left\langle c_{k}|\hat{\rho}| c_{k}\right\rangle\left\langle d_{k}|-| c_{k}\right\rangle\left\langle c_{k}|\hat{\rho}-\hat{\rho}| c_{k}\right\rangle\left\langle c_{k}\right|\right]$.

Upon substituting Eq. (1) into Eq. (2), the maser equation takes the form

$$
\begin{aligned}
& \frac{d \hat{\rho}}{d t}=g\left[\hat{\sigma}_{a}^{\dagger} \hat{a} \hat{\rho}-\hat{\rho} \hat{\sigma}_{a}^{\dagger} \hat{a}-\hat{a}^{\dagger} \hat{\sigma}_{a} \hat{\rho}+\hat{\rho} \hat{a}^{\dagger} \hat{\sigma}_{a}+\hat{\sigma}_{b}^{\dagger} \hat{b} \hat{\rho}-\right. \\
& \left.-\hat{\rho} \hat{\sigma}_{b}^{\dagger} \hat{b}-\hat{b}^{\dagger} \hat{\sigma}_{b} \hat{\rho}+\hat{\rho} \hat{b}^{\dagger} \hat{\sigma}_{b}\right]+ \\
& +\frac{\kappa}{2}\left[2 \hat{a} \hat{\rho} \hat{a}^{\dagger}-\hat{a}^{\dagger} \hat{a} \hat{\rho}-\hat{\rho} \hat{a}^{\dagger} \hat{a}+2 \hat{b} \hat{\rho} \hat{b}^{\dagger}-\hat{b}^{\dagger} \hat{b} \hat{\rho}-\hat{\rho} \hat{b}^{\dagger} \hat{b}\right]+ \\
& +\frac{\gamma}{2}\left[2\left|d_{k}\right\rangle\left\langle a_{k}|\hat{\rho}| a_{k}\right\rangle\left\langle d_{k}|-| a_{k}\right\rangle\left\langle a_{k}|\hat{\rho}-\hat{\rho}| a_{k}\right\rangle\left\langle a_{k}\right|+\right. \\
& +2\left|d_{k}\right\rangle\left\langle b_{k}|\hat{\rho}| b_{k}\right\rangle\left\langle d_{k}|-| b_{k}\right\rangle\left\langle b_{k}|\hat{\rho}-\hat{\rho}| b_{k}\right\rangle\left\langle b_{k}\right|+ \\
& \left.+2\left|d_{k}\right\rangle\left\langle c_{k}|\hat{\rho}| c_{k}\right\rangle\left\langle d_{k}|-| c_{k}\right\rangle\left\langle c_{k}|\hat{\rho}-\hat{\rho}| c_{k}\right\rangle\left\langle c_{k}\right|\right] .
\end{aligned}
$$

Here, the atomic coherences and atomic populations are defined by $\hat{\sigma}_{a}^{k}=\left|b_{k}\right\rangle\left\langle a_{k}\left|, \hat{\sigma}_{b}^{k}=\right| c_{k}\right\rangle\left\langle b_{k}\right|, \hat{\sigma}_{c}^{k}=$ $=\left|c_{k}\right\rangle\left\langle a_{k}\left|, \quad \hat{\eta}_{a}^{k}=\right| a_{k}\right\rangle\left\langle a_{k}\left|, \quad \hat{\eta}_{b}^{k}=\right| b_{k}\right\rangle\left\langle b_{k}\right|, \quad \hat{\eta}_{c}^{k}=$ $=\left|c_{k}\right\rangle\left\langle c_{K}\left|, \hat{\eta}_{d}^{k}=\right| d_{k}\right\rangle\left\langle d_{k}\right|$, respectively.
The equations of evolution for the expectation value of the cavity mode and atomic operators are evaluated by making use of the relation

$\frac{d}{d t}\langle\hat{A}\rangle=\operatorname{Tr}\left(\frac{d \hat{\rho}}{d t} \hat{A}\right)$

With regard for Eqs. (3) and (4), we can arrive at

$\frac{d}{d t}\langle\hat{a}\rangle=-\frac{\kappa}{2}\langle\hat{a}\rangle-g\left\langle\hat{\sigma}_{a}^{k}\right\rangle$,

$\frac{d}{d t}\langle\hat{b}\rangle=-\frac{\kappa}{2}\langle\hat{b}\rangle-g\left\langle\hat{\sigma}_{b}^{k}\right\rangle$,

$\frac{d}{d t}\left\langle\hat{\sigma}_{a}^{k}\right\rangle=-\gamma\left\langle\hat{\sigma}_{a}^{k}\right\rangle+g\left[\left\langle\hat{\eta}_{b}^{k} \hat{a}\right\rangle-\left\langle\hat{\eta}_{a}^{k} \hat{a}\right\rangle+\left\langle\hat{b}^{\dagger} \hat{\sigma}_{c}^{k}\right\rangle\right]$

$\frac{d}{d t}\left\langle\hat{\sigma}_{b}^{k}\right\rangle=-\gamma\left\langle\hat{\sigma}_{b}^{k}\right\rangle+g\left[\left\langle\hat{\eta}_{c}^{k} \hat{b}\right\rangle-\left\langle\hat{a}^{\dagger} \hat{\sigma}_{c}^{k}\right\rangle-\left\langle\hat{\eta}_{b}^{k} \hat{b}\right\rangle\right]$,

$\frac{d}{d t}\left\langle\hat{\sigma}_{c}^{k}\right\rangle=-\gamma\left\langle\hat{\sigma}_{c}^{k}\right\rangle+g\left[\left\langle\hat{\sigma}_{b}^{k} \hat{a}\right\rangle-\left\langle\hat{\sigma}_{a}^{k} \hat{b}\right\rangle\right]$

$\frac{d}{d t}\left\langle\hat{\eta}_{a}^{k}\right\rangle=-\gamma\left\langle\hat{\eta}_{a}^{k}\right\rangle+g\left[\left\langle\hat{\sigma}_{a}^{\dagger k} \hat{a}\right\rangle+\left\langle\hat{a}^{\dagger} \hat{\sigma}_{a}^{k}\right\rangle\right]$,

$\frac{d}{d t}\left\langle\hat{\eta}_{b}^{k}\right\rangle=\gamma\left(\left\langle\hat{\eta}_{a}^{k}\right\rangle-\left\langle\hat{\eta}_{b}^{k}\right\rangle\right)-g\left[\left\langle\hat{\sigma}_{a}^{\dagger k} \hat{a}\right\rangle+\left\langle\hat{a}^{\dagger k} \hat{\sigma}_{a}\right\rangle\right]$,

$\frac{d}{d t}\left\langle\hat{\eta}_{c}^{k}\right\rangle=-\gamma\left\langle\hat{\eta}_{c}^{k}\right\rangle-g\left[\left\langle\hat{\sigma}_{b}^{\dagger k} \hat{b}\right\rangle+\left\langle\hat{b}^{\dagger} \hat{\sigma}_{b}^{k}\right\rangle\right]$

$\frac{d}{d t}\left\langle\hat{\eta}_{d}^{k}\right\rangle=\gamma\left[\left\langle\hat{\eta}_{a}^{k}\right\rangle+\left\langle\hat{\eta}_{b}^{k}\right\rangle+\left\langle\hat{\eta}_{c}^{k}\right\rangle\right]$

On the basis of expressions (5) and (6), we can rewrite

$\frac{d}{d t}\langle\hat{a}\rangle=-\frac{\kappa}{2} \hat{a}-g \hat{\sigma}_{a}^{k}+\hat{g}_{a}(t)$

and

$\frac{d}{d t}\langle\hat{b}\rangle=-\frac{\kappa}{2} \hat{b}-g \hat{\sigma}_{b}^{k}+\hat{g}_{b}(t)$

where $\hat{g}_{a}(t)$ and $\hat{g}_{b}(t)$ are the quantum Langevin noise operators associated with the cavity mode, whose correlation properties are expressible as [16]

$\left\langle\hat{g}_{a}(t)\right\rangle=\left\langle\hat{g}_{b}(t)\right\rangle=0$,

$\left\langle\hat{g}_{a}^{\dagger}(t) \hat{g}_{a}\left(t^{\prime}\right)\right\rangle=\left\langle\hat{g}_{b}^{\dagger}(t) \hat{g}_{b}\left(t^{\prime}\right)\right\rangle=0$,

$\left\langle\hat{g}_{a}(t) \hat{g}_{a}^{\dagger}\left(t^{\prime}\right)\right\rangle=\left\langle\hat{g}_{b}(t) \hat{g}_{b}^{\dagger}\left(t^{\prime}\right)\right\rangle=\kappa \delta\left(t-t^{\prime}\right)$,

$\left\langle\hat{g}_{a}^{\dagger}(t) \hat{g}_{a}^{\dagger}\left(t^{\prime}\right)\right\rangle=\left\langle\hat{g}_{a}(t) \hat{g}_{a}\left(t^{\prime}\right)\right\rangle=\left\langle\hat{g}_{b}(t) \hat{g}_{b}\left(t^{\prime}\right)\right\rangle=0$.

We see that Eqs. (7)-(13) are incomplete linear coupled differential equations. Hence, it is not possible

ISSN 2071-0194. Ukr. J. Phys. 2021. Vol. 66, No. 3 
to find exact time-dependent solutions for these equations [17]. To overcome this problem, we consider the case where the cavity mode operators relax firstly [1620]. Then, in view of this consideration, Eqs. (14) and (15) yield the following approximately valid relations:

$\hat{a}=-\frac{2 g}{\kappa} \hat{\sigma}_{a}^{k}+\frac{2}{\kappa} \hat{g}_{a}(t)$,

$\hat{b}=-\frac{2 g}{\kappa} \hat{\sigma}_{b}^{k}+\frac{2}{\kappa} \hat{g}_{b}(t)$.

Then, substituting (20) and (21) into Eqs. (7)-(13), we obtain

$\frac{d}{d t}\left\langle\hat{\sigma}_{a}^{k}(t)\right\rangle=-\left(\gamma+\gamma_{c}\right)\left\langle\hat{\sigma}_{a}^{k}(t)\right\rangle+\frac{2 g}{\kappa}\left[\left\langle\hat{\eta}_{b}^{k}(t) \hat{g}_{a}(t)\right\rangle-\right.$

$\left.-\left\langle\hat{\eta}_{a}^{k}(t) \hat{g}_{a}(t)\right\rangle+\left\langle g_{a}^{\dagger k}(t) \hat{\sigma}_{c}^{k}(t)\right\rangle\right]$,

$\frac{d}{d t}\left\langle\hat{\sigma}_{b}^{k}(t)\right\rangle=-\left(\frac{\gamma_{c}}{2}+\gamma\right)\left\langle\hat{\sigma}_{b}^{k}(t)\right\rangle+\frac{2 g}{\kappa}\left[\left\langle\hat{\eta}_{c}^{k}(t) \hat{g}_{b}(t)\right\rangle-\right.$

$\left.-\left\langle\hat{g}_{a}(t) \hat{\sigma}_{c}^{k}(t)\right\rangle-\left\langle\hat{\eta}_{b}^{k}(t) \hat{g}_{b}(t)\right\rangle\right]$

$\frac{d}{d t}\left\langle\hat{\sigma}_{c}^{k}(t)\right\rangle=-\left(\frac{\gamma_{c}}{2}+\gamma\right)\left\langle\hat{\sigma}_{c}^{k}(t)\right\rangle+$

$+\frac{2 g}{\kappa}\left[\left\langle\hat{\sigma}_{a}^{k}(t) \hat{g}_{b}(t)\right\rangle-\left\langle\hat{\sigma}_{b}^{k}(t) \hat{g}_{a}(t)\right\rangle\right]$,

$\frac{d}{d t}\left\langle\hat{\eta}_{a}^{k}(t)\right\rangle=-\left(\gamma_{c}+\gamma\right)\left\langle\hat{\eta}_{a}^{k}(t)\right\rangle+$

$+\frac{2 g}{\kappa}\left[\left\langle\hat{\sigma}_{a}^{\dagger k}(t) \hat{g}_{a}(t)\right\rangle+\left\langle\hat{g}_{a}^{\dagger}(t) \hat{\sigma}_{a}^{k}(t)\right\rangle\right]$,

$\frac{d}{d t}\left\langle\hat{\eta}_{b}^{k}(t)\right\rangle=-\left(\gamma+\gamma_{c}\left\langle\hat{\eta}_{b}(t)\right\rangle+\gamma_{c}\left\langle\hat{\eta}_{a}(t)\right\rangle+\right.$

$+\frac{2 g}{\kappa}\left[\left\langle\hat{\sigma}_{a}^{\dagger k}(t) \hat{g}_{a}(t)\right\rangle+\left\langle\hat{g}_{a}^{\dagger}(t) \hat{\sigma}_{a}^{k}(t)\right\rangle\right]$,

$\frac{d}{d t}\left\langle\hat{\eta}_{c}^{k}(t)\right\rangle=-\gamma\left\langle\hat{\eta}_{c}^{k}\right\rangle+\gamma_{c}\left\langle\hat{\eta}_{a}(t)\right\rangle-$

$-\frac{2 g}{\kappa}\left[\left\langle\hat{g}_{b}^{\dagger}(t) \hat{\sigma}_{b}^{k}(t)\right\rangle+\left\langle\hat{\sigma}_{b}^{\dagger k}(t) \hat{g}_{b}(t)\right\rangle\right]$

where

$\gamma_{c}=\frac{4 g^{2}}{\kappa}$

is the rate of stimulated emission decay [6].

Next, we wish to determine the values of the terms inside the square brackets in (7). To this end, after ISSN 2071-0194. Ukr. J. Phys. 2021. Vol. 66, No. 3 removing the angular brackets, the formal solution of Eq. (10) can be written as

$\hat{\eta}_{a}^{k}(t)=\hat{\eta}_{a}^{k}(0) e^{-\left(\gamma+\gamma_{c}\right) t}+\frac{2 g}{\kappa} \int_{0}^{t} d t^{\prime} e^{-\left(\gamma+\gamma_{c}\right)\left(t-t^{\prime}\right)} \times$

$\times\left[\hat{\sigma}_{a}^{\dagger k}\left(t^{\prime}\right) \hat{g}_{a}\left(t^{\prime}\right)+\hat{g}_{a}^{\dagger}\left(t^{\prime}\right) \hat{\sigma}_{a}^{k}\left(t^{\prime}\right)\right]+$

$+\int_{0}^{t} d t^{\prime} e^{-\left(\gamma+\gamma_{c}\right)\left(t-t^{\prime}\right)} \hat{F}_{a}\left(t^{\prime}\right)$

where $\hat{F}_{a}(t)$ is a vacuum reservoir noise operator associated with an atomic operator. Now, multiplying the above equation from the right by $\hat{g}_{a}(t)$ and taking the expectation value of the resulting expression, we get

$\left\langle\hat{\eta}_{a}^{k}(t) \hat{g}_{a}(t)\right\rangle=\left\langle\hat{\eta}_{a}^{k}(0) \hat{g}_{a}(t)\right\rangle e^{-\left(\gamma+\gamma_{c}\right) t}+$

$+\int_{0}^{t} d t^{\prime} e^{-\left(\gamma+\gamma_{c}\right)\left(t-t^{\prime}\right)}\left[\frac{2 g}{\kappa}\left\{\left\langle\hat{\sigma}_{a}^{\dagger k}\left(t^{\prime}\right) \hat{g}_{a}\left(t^{\prime}\right) \hat{g}_{a}(t)\right\rangle+\right.\right.$

$\left.\left.+\left\langle\hat{g}_{a}^{\dagger}\left(t^{\prime}\right) \hat{\sigma}_{a}^{k}\left(t^{\prime}\right) \hat{g}_{a}(t)\right\rangle\right\}+\left\langle\hat{F}_{a}\left(t^{\prime}\right) \hat{g}_{a}(t)\right\rangle\right]$.

Accounting for the fact that the noise operator $\hat{g}_{a}(t)$ at a certain time does not affect the atomic operator at earlier times and assuming that there is no correlation between the vacuum reservoir noise operators associated with the atomic and cavity mode operators, Eq. (30) can be put in the form

$$
\begin{aligned}
& \left\langle\hat{\eta}_{a}^{k}(t) \hat{g}_{a}(t)\right\rangle=\frac{2 g}{\kappa} \int_{0}^{t} d t^{\prime} e^{-\left(\gamma+\gamma_{c}\right)\left(t-t^{\prime}\right)} \times \\
& \times\left[\left\langle\hat{\sigma}_{a}^{\dagger k}\left(t^{\prime}\right) \hat{g}_{a}\left(t^{\prime}\right) \hat{g}_{a}(t)\right\rangle+\left\langle\hat{g}_{a}^{\dagger}\left(t^{\prime}\right) \hat{\sigma}_{a}^{k}\left(t^{\prime}\right) \hat{g}_{a}(t)\right\rangle\right] .
\end{aligned}
$$

It is impossible to evaluate the integral that appears in Eq. (31), since the explicit form of $\hat{\sigma}_{a}^{k}\left(t^{\prime}\right)$ is unknown yet. In order to proceed further, we need to adopt a certain approximation scheme. To this end, ignoring the noncommutativity of the atomic and noise operators $[16,18]$, we see that

$\left\langle\hat{g}_{a}^{\dagger}\left(t^{\prime}\right) \hat{\sigma}_{a}^{k}\left(t^{\prime}\right) \hat{g}_{a}(t)\right\rangle=\left\langle\hat{\sigma}_{a}^{k}\left(t^{\prime}\right) \hat{g}_{a}^{\dagger}\left(t^{\prime}\right) \hat{g}_{a}(t)\right\rangle$.

Then, upon neglecting the correlation between $\hat{\sigma}_{a}^{k}\left(t^{\prime}\right)$ and $\hat{g}_{a}^{\dagger}\left(t^{\prime}\right) \hat{g}_{a}(t)$, we can write the approximately valid relation

$\left\langle\hat{\sigma}_{a}^{k}\left(t^{\prime}\right) \hat{g}_{a}^{\dagger}\left(t^{\prime}\right) \hat{g}_{a}(t)\right\rangle=\left\langle\hat{\sigma}_{a}^{k}\left(t^{\prime}\right)\right\rangle\left\langle\hat{g}_{a}^{\dagger}\left(t^{\prime}\right) \hat{g}_{a}(t)\right\rangle$. 
In view of Eq. (17), Eq. (33) takes the form

$\left\langle\hat{\sigma}_{a}^{k}\left(t^{\prime}\right) \hat{g}_{a}^{\dagger}\left(t^{\prime}\right) \hat{g}_{a}(t)\right\rangle=0$.

Again, by neglecting the correlation between $\hat{\sigma}_{a}^{\dagger k}\left(t^{\prime}\right)$ and $\hat{g}_{a}\left(t^{\prime}\right) \hat{g}_{a}(t)$, we can write

$\left\langle\hat{\sigma}_{a}^{\dagger k}\left(t^{\prime}\right) \hat{g}_{a}\left(t^{\prime}\right) \hat{g}_{a}(t)\right\rangle=\left\langle\hat{\sigma}_{a}^{\dagger k}\left(t^{\prime}\right)\right\rangle\left\langle\hat{g}_{a}\left(t^{\prime}\right) \hat{g}_{a}(t)\right\rangle$.

Now, in view of Eq. (19), the above expression turns out to be

$\left\langle\hat{\sigma}_{a}^{\dagger k}\left(t^{\prime}\right) \hat{g}_{a}\left(t^{\prime}\right) \hat{g}_{a}(t)\right\rangle=0$.

Hence, on the basis of Eqs. (34) and (36), Eq. (31) can be put in the form

$\left\langle\hat{\eta}_{a}^{k}(t) \hat{g}_{a}(t)\right\rangle=0$.

Following a similar line of reasoning, we can also easily verify that

$\left\langle\hat{\eta}_{b}^{k}(t) \hat{g}_{a}(t)\right\rangle=0$,

$\left\langle\hat{g}_{b}^{\dagger k}(t) \hat{\sigma}_{c}^{k}(t)\right\rangle=0$.

In view of Eqs. (37), (38), and (39), Eq. (7) takes the form

$\frac{d}{d t}\left\langle\hat{\sigma}_{a}^{k}(t)\right\rangle=-\left(\gamma+\gamma_{c}\right)\left\langle\hat{\sigma}_{a}^{k}(t)\right\rangle$.

It can also be readily established in a similar manner that

$\frac{d}{d t}\left\langle\hat{\sigma}_{b}^{k}(t)\right\rangle=-\left(\frac{\gamma_{c}}{2}+\gamma\right)\left\langle\hat{\sigma}_{b}^{k}(t)\right\rangle$,

$\frac{d}{d t}\left\langle\hat{\sigma}_{c}^{k}(t)\right\rangle=-\left(\frac{\gamma_{c}}{2}+\gamma\right)\left\langle\hat{\sigma}_{c}^{k}(t)\right\rangle$,

$\frac{d}{d t}\left\langle\hat{\eta}_{a}^{k}(t)\right\rangle=-\left(\gamma_{c}+\gamma\right)\left\langle\hat{\eta}_{a}^{k}(t)\right\rangle$,

$\frac{d}{d t}\left\langle\hat{\eta}_{b}^{k}(t)\right\rangle=-\left(\gamma+\gamma_{c}\right)\left\langle\hat{\eta}_{b}(t)\right\rangle+\gamma_{c}\left\langle\hat{\eta}_{a}(t)\right\rangle$,

$\frac{d}{d t}\left\langle\hat{\eta}_{c}^{k}(t)\right\rangle=-\gamma\left\langle\hat{\eta}_{c}^{k}\right\rangle+\gamma_{c}\left\langle\hat{\eta}_{a}(t)\right\rangle$.

In order to include the contribution of all the atoms to the dynamics of the three-level laser, we sum Eqs. (40)-(45) over the $N$ three-level atoms, so that

$\frac{d}{d t}\left\langle\hat{m}_{a}(t)\right\rangle=-\left(\gamma_{c}+\gamma\right)\left\langle\hat{m}_{a}(t)\right\rangle$,

210

$$
\begin{aligned}
\frac{d}{d t}\left\langle\hat{m}_{b}(t)\right\rangle & =-\left(\frac{\gamma_{c}}{2}+\gamma\right)\left\langle\hat{m}_{b}(t)\right\rangle, \\
\frac{d}{d t}\left\langle\hat{m}_{c}(t)\right\rangle & =-\left(\frac{\gamma_{c}}{2}+\gamma\right)\left\langle\hat{m}_{c}(t)\right\rangle, \\
\frac{d}{d t}\left\langle\hat{N}_{a}(t)\right\rangle & =-\left(\gamma_{c}+\gamma\right)\left\langle\hat{N}_{a}(t)\right\rangle, \\
\frac{d}{d t}\left\langle\hat{N}_{b}(t)\right\rangle & =-\left(\gamma+\gamma_{c}\right)\left\langle\hat{N}_{b}(t)\right\rangle+\gamma_{c}\left\langle\hat{N}_{a}(t)\right\rangle, \\
\frac{d}{d t}\left\langle\hat{N}_{c}(t)\right\rangle & =-\gamma\left\langle\hat{N}_{c}(t)\right\rangle+\gamma_{c}\left\langle\hat{N}_{a}(t)\right\rangle, \\
\frac{d}{d t}\left\langle\hat{N}_{d}(t)\right\rangle & =\gamma\left[\left\langle\hat{N}_{a}(t)\right\rangle+\left\langle\hat{N}_{b}(t)\right\rangle+\left\langle\hat{N}_{c}(t)\right\rangle\right],
\end{aligned}
$$

where

$$
\begin{aligned}
& \hat{m}_{i}=\sum_{k=1}^{N} \hat{\sigma}_{i}^{k}=N \hat{\sigma}_{i}, \\
& \hat{N}_{j}=\sum_{k=1}^{N} \hat{\eta}_{j}^{k}=N \hat{\eta}_{j},
\end{aligned}
$$

in which $i=a, b, c$ and $j=a, b, c, d$ and $\hat{N}_{a}, \hat{N}_{b}, \hat{N}_{c}$, and $\hat{N}_{d}$ are atomic operators representing the number of atoms in the upper, intermediate, bottom, and ground levels, respectively.

Furthermore, the three-level atoms available in the cavity are pumped from the ground and the bottom levels to the top level by means of the electron bombardment. The pumping process must surely affect the dynamics of $\left\langle\hat{N}_{a}\right\rangle,\left\langle\hat{N}_{c}\right\rangle$, and $\left\langle\hat{N}_{d}\right\rangle$. Then $\left\langle\hat{N}_{a}\right\rangle$ increases at the rates of $r_{a}\left\langle\hat{N}_{c}\right\rangle$ and $r_{a}\left\langle\hat{N}_{d}\right\rangle$ where as $\left\langle\hat{N}_{c}\right\rangle$ and $\left\langle\hat{N}_{d}\right\rangle$ decreases at the rates of $r_{a}\left\langle\hat{N}_{c}\right\rangle$ and $r_{a}\left\langle\hat{N}_{d}\right\rangle$, respectively [12]. Here, $r_{a}$, assumed to be the same for all transitions, represents the rate at which a single atom is pumped from the bottom and ground levels to the top level.

Incorporating the effect of the pumping process, Eqs. (49), (51), and (52) become

$$
\begin{aligned}
& \frac{d}{d t}\left\langle\hat{N}_{a}\right\rangle=-\left[\gamma_{c}+\gamma\right]\left\langle\hat{N}_{a}\right\rangle+r_{a}\left[\left\langle\hat{N}_{c}\right\rangle+\left\langle\hat{N}_{d}\right\rangle\right], \\
& \frac{d}{d t}\left\langle\hat{N}_{c}(t)\right\rangle=-\left[\gamma+r_{a}\right]\left\langle\hat{N}_{c}(t)\right\rangle+\gamma_{c}\left\langle\hat{N}_{a}(t)\right\rangle, \\
& \frac{d}{d t}\left\langle\hat{N}_{d}\right\rangle=-r_{a}\left\langle\hat{N}_{d}\right\rangle+\gamma\left[\left\langle\hat{N}_{a}\right\rangle+\left\langle\hat{N}_{b}\right\rangle+\left\langle\hat{N}_{c}\right\rangle\right] .
\end{aligned}
$$

The steady-state solutions for the numbers of atoms in the upper, intermediate, bottom, and ground levels are expressible as

$\left\langle\hat{N}_{a}\right\rangle=\left[\frac{\left\langle\hat{N}_{c}\right\rangle+\left\langle\hat{N}_{d}\right\rangle}{\gamma+\gamma_{c}}\right] r_{a}$

ISSN 2071-0194. Ukr. J. Phys. 2021. Vol. 66, No. 3 
$\left\langle\hat{N}_{b}\right\rangle=\left[\frac{\gamma_{c}}{\gamma+\gamma_{c}}\right]\left\langle\hat{N}_{a}\right\rangle$,

$\left\langle\hat{N}_{c}\right\rangle=\left[\frac{\gamma_{c}}{\gamma+r_{a}}\right]\left\langle\hat{N}_{a}\right\rangle$,

$\left\langle\hat{N}_{d}\right\rangle=\frac{\gamma}{r_{a}}\left[\left\langle\hat{N}_{a}\right\rangle+\left\langle\hat{N}_{b}\right\rangle+\left\langle\hat{N}_{c}\right\rangle\right]$.

Applying the completeness relation

$\hat{\eta}_{a}^{k}+\hat{\eta}_{b}^{k}+\hat{\eta}_{c}^{k}+\hat{\eta}_{d}^{k}=1$,

we can easily write

$\left\langle\hat{N}_{a}\right\rangle+\left\langle\hat{N}_{b}\right\rangle+\left\langle\hat{N}_{c}\right\rangle+\left\langle\hat{N}_{d}\right\rangle=N$.

Making use of this expression, Eq. (61) can be rewritten as

$\left\langle\hat{N}_{d}\right\rangle=\left[\frac{\gamma}{r_{a}+\gamma}\right] N$.

In view of Eqs. (60) and (64), Eq. (58) becomes

$\left\langle\hat{N}_{a}\right\rangle=\left[\frac{r_{a}\left(\gamma+\gamma_{c}\right)}{\left(\gamma+\gamma_{c}\right)^{2}+r_{a}\left(\gamma+2 \gamma_{c}\right)}\right] N$.

Using Eq. (65), Eqs. (59) and (60) become

$\left\langle\hat{N}_{b}\right\rangle=\left[\frac{r_{a} \gamma_{c}}{\left(\gamma+\gamma_{c}\right)^{2}+r_{a}\left(\gamma+2 \gamma_{c}\right)}\right] N$

and

$\left\langle\hat{N}_{c}\right\rangle=\left[\frac{r_{a} \gamma_{c}^{2}}{\left[\gamma+r_{a}\right]\left[\left(\gamma+\gamma_{c}\right)^{2}+r_{a}\left(\gamma+2 \gamma_{c}\right)\right]}\right] N$.

On the other hand, when the cavity modes are interacting with all three-level atoms available in the cavity, following the same line of reasoning conducted by Mekonnen [6], we can rewrite Eqs. (5) and (6) as

$\frac{d}{d t} \hat{a}(t)=-\frac{\kappa}{2} \hat{a}(t)+\frac{g}{\sqrt{N}} \hat{m}_{a}(t)+\hat{g}_{a}(t)$

and

$\frac{d}{d t} \hat{b}(t)=-\frac{\kappa}{2} \hat{b}(t)+\frac{g}{\sqrt{N}} \hat{m}_{b}(t)+\hat{g}_{b}(t)$.

The formal solution of (73) can be expressed as

$$
\begin{aligned}
& \hat{a}(t)=a(0) e^{-\frac{\kappa}{2} t}+ \\
& +\int_{0}^{t} d t^{\prime} e^{-\frac{\kappa}{2}\left(t-t^{\prime}\right)}\left[\frac{g}{\sqrt{N}} \hat{m}_{a}\left(t^{\prime}\right)+\hat{g}_{a}\left(t^{\prime}\right)\right] .
\end{aligned}
$$

ISSN 2071-0194. Ukr. J. Phys. 2021. Vol. 66, No. 3
Moreover, the solution of (46) can be expressible as $\hat{m}_{a}(t)=m_{a}(0) e^{-\left(\gamma+\gamma_{c}\right) t}+\int_{0}^{t} d t^{\prime} e^{-\left(\gamma+\gamma_{c}\right)\left(t-t^{\prime}\right)} \hat{q}\left(t^{\prime}\right)$,

where $\hat{q}(t)$ is the Langevin noise operator associated with the atom. Now, accounting for the correlation property $\langle\hat{q}(t)\rangle=0$ and assuming that the three-level atoms are initially in the bottom level, the expectation value of this equation is found to be

$\left\langle\hat{m}_{a}(t)\right\rangle=0$.

Furthermore, using (72) and the assumption that the cavity modes are initially in a vacuum state along with (16), one obtains from (70):

$\langle\hat{a}(t)\rangle=0$.

It can also be verified in a similar manner that

$\langle\hat{b}(t)\rangle=0$.

Therefore, we see from Eqs. (68) and (73), as well as from (69) and (74), that $\hat{a}(t)$ and $\hat{b}(t)$ are Gaussian variables with the vanishing mean [19]. Moreover, adding Eq. (73) and (74), we find

$\frac{d \hat{c}(t)}{d t}=-\frac{\kappa}{2} \hat{c}(t)+\frac{g}{\sqrt{N}} \hat{m}(t)+\hat{F}_{c}(t)$,

where $\hat{c}=\hat{a}+\hat{b}, \hat{m}=\hat{m}_{a}+\hat{m}_{b}, \hat{F}_{c}=\hat{F}_{a}+\hat{F}_{b}$.

\section{Photon Statistics}

Here, we will study the statistical properties of the light modes produced by an electrically driven threelevel laser with an open cavity coupled to a two-mode vacuum reservoir via a single port mirror. Applying the steady-state solutions of the equations of evolution of the expectation values of the atomic operators and the quantum Langevin equations, we obtain the global and local photon statistics of the single- and two-mode light beams.

\subsection{The global mean photon number}

The mean photon number for light mode $a$ at any time $t$ is given by $\left\langle\hat{a}^{\dagger}(t) \hat{a}(t)\right\rangle$ (see Fig. 1). The equation of evolution of the mean photon number is expressible as [15]

$\frac{d}{d t}\left\langle\hat{a}^{\dagger} \hat{a}\right\rangle=\left\langle\frac{d \hat{a}^{\dagger}}{d t} \hat{a}\right\rangle+\left\langle\hat{a}^{\dagger} \frac{d \hat{a}}{d t}\right\rangle$. 


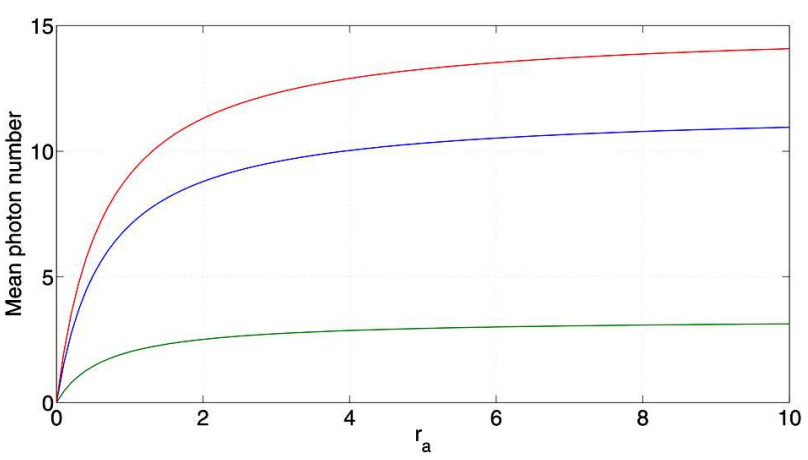

Fig. 1. Plot of the mean photon number for light modes $a, b$ and $c$ versus $r_{a}$ for $\kappa=0.8, \gamma=0.5, \gamma_{c}=0.4$, and $N=50$

Employing (68) and its adjoint, Eq. (76) becomes

$\frac{d}{d t}\left\langle\hat{a}^{\dagger} \hat{a}\right\rangle=-\kappa\left\langle\hat{a}^{\dagger} \hat{a}\right\rangle+\frac{4}{\kappa} \frac{g^{2}}{N}\left\langle\hat{m}_{a}^{\dagger} \hat{m}_{a}\right\rangle$.

The solution of this expression in the steady state reduces to

$\left\langle\hat{a}^{\dagger} \hat{a}\right\rangle=\frac{4}{\kappa^{2}} \frac{g^{2}}{N}\left\langle\hat{m}_{a}^{\dagger} \hat{m}_{a}\right\rangle$

With regard for (28) and the relation $\left\langle\hat{m}_{a}^{\dagger} \hat{m}_{a}\right\rangle=$ $=N\left\langle\hat{N}_{a}\right\rangle$, the above expression takes the form

$\left\langle\hat{a}^{\dagger} \hat{a}\right\rangle=\frac{\gamma_{c}}{\kappa}\left\langle\hat{N}_{a}\right\rangle$

Introducing Eq. (65) into this expression, we obtain

$\bar{n}_{a}=\frac{\gamma_{c}}{\kappa}\left[\frac{r_{a}\left(\gamma+\gamma_{c}\right)}{\left(\gamma+\gamma_{c}\right)^{2}+r_{a}\left(\gamma+2 \gamma_{c}\right)}\right] N$.

Following a similar procedure, we get the relation for the mean photon number of light mode $b$

$\bar{n}_{b}=\frac{\gamma_{c}}{\kappa}\left[\frac{r_{a} \gamma_{c}}{\left(\gamma+\gamma_{c}\right)^{2}+r_{a}\left(\gamma+2 \gamma_{c}\right)}\right] N$

and

$\bar{n}_{c}=\frac{\gamma_{c}}{\kappa}\left[\frac{r_{a}\left(\gamma+2 \gamma_{c}\right)}{\left(\gamma+\gamma_{c}\right)^{2}+r_{a}\left(\gamma+2 \gamma_{c}\right)}\right] N$.

From Eqs. (80) and (81), it can be easily observed that $\bar{n}_{a}=\bar{n}_{b}$ in the absence of spontaneous emission. In addition, comparing and contrasting the above three consecutive expressions, we conclude that the mean photon number of the two-mode cavity light is just the sum of the mean photon numbers of the separate single-mode cavity light.

\subsection{The local mean photon number}

Next, we calculate the mean photon number of single and two-mode lights in a given frequency interval. To do so, we need first to determine the power spectrum of light modes $a, b$, and $c$. To this end, the power spectrum of light mode $a$ with the central frequency $\omega_{0}$ is given by [3]

$P_{a}(\omega)=\frac{1}{\pi} \operatorname{Re} \int_{0}^{\infty} d \tau e^{i\left(\omega-\omega_{0}\right) \tau}\left\langle\hat{a}^{\dagger}(t) \hat{a}(t+\tau)\right\rangle_{s s}$,

where $s s$ refers to the steady state. Now, by integrating this expression over the whole frequency interval, we arrive at

$\int_{-\infty}^{\infty} P_{a}(\omega) d \omega=\bar{n}_{a}$

where $\bar{n}_{a}$ is the steady-state mean photon number of light mode $a$ [3]. From this result, we observe that $P_{a}(\omega) d \omega$ is the steady-state mean photon number of light mode $a$ in the frequency interval between $\omega$ and $\omega+d \omega[3]$.

We now proceed to calculate the two-time correlation function that appears in (83). To this end, we realize that the two-time correlation function that appears in (83) is found to be

$$
\begin{aligned}
& \left\langle\hat{a}^{\dagger}(t) \hat{a}(t+\tau)\right\rangle_{s s}=\bar{n}_{a}\left[\frac{\kappa}{\kappa-2\left(\gamma+\gamma_{c}\right)} e^{-\left(\gamma+\gamma_{c}\right) \tau}-\right. \\
& \left.-\frac{2\left(\gamma+\gamma_{c}\right)}{\kappa-2\left(\gamma+\gamma_{c}\right)} e^{-\frac{1}{2} \kappa \tau}\right] .
\end{aligned}
$$

Now, by substituting Eq. (85) into (83), we get the power spectrum of light mode $a$ in the form

$$
\begin{aligned}
& P_{a}(\omega)=\frac{\kappa \bar{n}_{a}}{\kappa-2\left(\gamma+\gamma_{c}\right)}\left[\frac{\left(\gamma+\gamma_{c}\right) / \pi}{\left(\omega-\omega_{0}\right)^{2}+\left(\gamma+\gamma_{c}\right)^{2}}\right]- \\
& -\frac{2\left(\gamma+\gamma_{c}\right) \bar{n}_{a}}{\kappa-2\left(\gamma+\gamma_{c}\right)}\left[\frac{\kappa / 2 \pi}{\left(\omega-\omega_{0}\right)^{2}+\left(\frac{\kappa}{2}\right)^{2}}\right] .
\end{aligned}
$$

We recall that the mean photon number of light mode $a$ in the interval between $\omega^{\prime}=-\lambda$ and $\omega^{\prime}=\lambda$ is expressible as [19]

$\bar{n}_{a \pm \lambda}=\int_{-\lambda}^{\lambda} P_{a}\left(\omega^{\prime}\right) d \omega^{\prime}$

ISSN 2071-0194. Ukr. J. Phys. 2021. Vol. 66, No. 3 
where $\omega^{\prime}=\omega-\omega_{0}$. Therefore, by substituting (86) into (87), carrying out the integration, and making use of the relation

$\int_{-\lambda}^{\lambda} \frac{d x}{x^{2}+a^{2}}=\frac{2}{a} \tan ^{-1}\left(\frac{\lambda}{a}\right)$,

we arrive at

$\bar{n}_{a \pm \lambda}=\bar{n}_{a}\left[\frac{2 \kappa / \pi}{\kappa-2\left(\gamma+\gamma_{c}\right)} \tan ^{-1}\left(\frac{\lambda}{\gamma+\gamma_{c}}\right)-\right.$

$\left.-\frac{4\left(\gamma+\gamma_{c}\right) / \pi}{\kappa-2\left(\gamma+\gamma_{c}\right)} \tan ^{-1}\left(\frac{2 \lambda}{\kappa}\right)\right]$.

Now, the mean photon number of light mode $a$ in the frequency interval $\pm \lambda$ can be written as

$\bar{n}_{a \pm \lambda}=\bar{n}_{a} z_{a}(\lambda)$

where the characteristics function $z_{a}(\lambda)$ is given by

$z_{a}(\lambda)=\frac{2}{\pi}\left[\frac{\kappa}{\kappa-2\left(\gamma+\gamma_{c}\right)} \tan ^{-1}\left(\frac{\lambda}{\gamma+\gamma_{c}}\right)-\right.$

$\left.-\frac{2\left(\gamma+\gamma_{c}\right)}{\kappa-2\left(\gamma+\gamma_{c}\right)} \tan ^{-1}\left(\frac{2 \lambda}{\kappa}\right)\right]$.

We see from Eq. (90) and the plot of $z_{a}(\lambda)$ that $\bar{n}_{a \pm \lambda}$ increases with $\lambda$, until it reaches the maximum value given by Eq. (80). From the plot of Fig. 2, some of the values of $z_{a}(\lambda)$ and the corresponding local mean photon number $\bar{n}_{a \pm \lambda}$ are shown below.

Following a similar procedure, we can show that the mean photon number of light mode $b$ in the frequency interval $\pm \lambda$ is

$\bar{n}_{b \pm \lambda}=\bar{n}_{b} z_{b}(\lambda)$

where $z_{b}(\lambda)$ is given by

$z_{b}(\lambda)=\frac{2}{\pi}\left[\frac{\kappa}{\kappa-\left(2 \gamma+\gamma_{c}\right)} \tan ^{-1}\left(\frac{2 \lambda}{2 \gamma+\gamma_{c}}\right)-\right.$
$\left.-\frac{\left(2 \gamma+\gamma_{c}\right)}{\kappa-\left(2 \gamma+\gamma_{c}\right)} \tan ^{-1}\left(\frac{2 \lambda}{\kappa}\right)\right]$.

We see from Eq. (93) and the plot of $z_{b}(\lambda)$ that $\bar{n}_{b \pm \lambda}$ increases with $\lambda$, until it reaches the maximum value given by Eq. (81).

From Figs. 3 and 4 and Tables 1 and 2, we observe that a large part of the total mean photon number is ISSN 2071-0194. Ukr. J. Phys. 2021. Vol. 66, No. 3

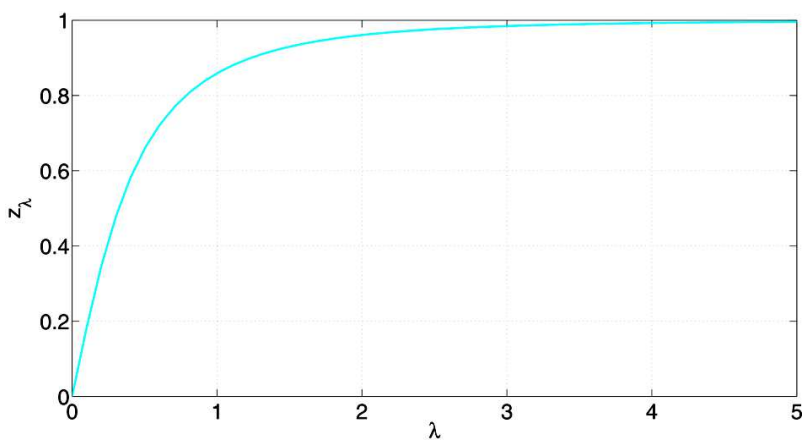

Fig. 2. Plot of $z_{a}(\lambda)$ [Eq. (91)] versus $\lambda$ for $\gamma_{c}=0.4, \kappa=0.8$, and $\gamma=2$

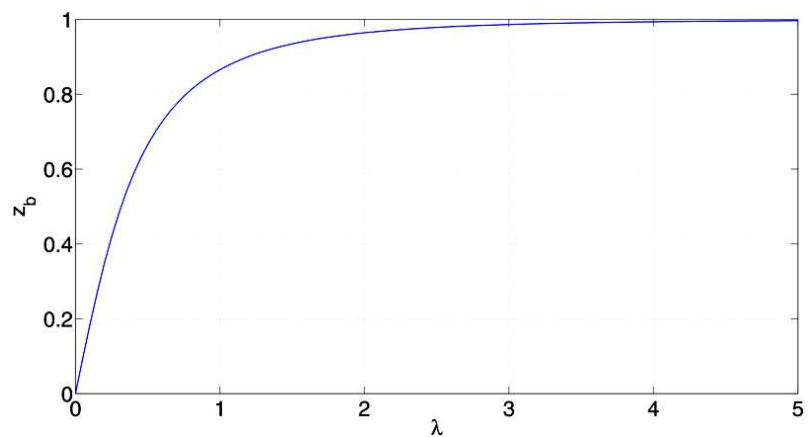

Fig. 3. Plot of $z_{b}(\lambda)$ [Eq. (93)] versus $\lambda$ for $\gamma_{c}=0.4, \kappa=0.8$, and $\gamma=2$

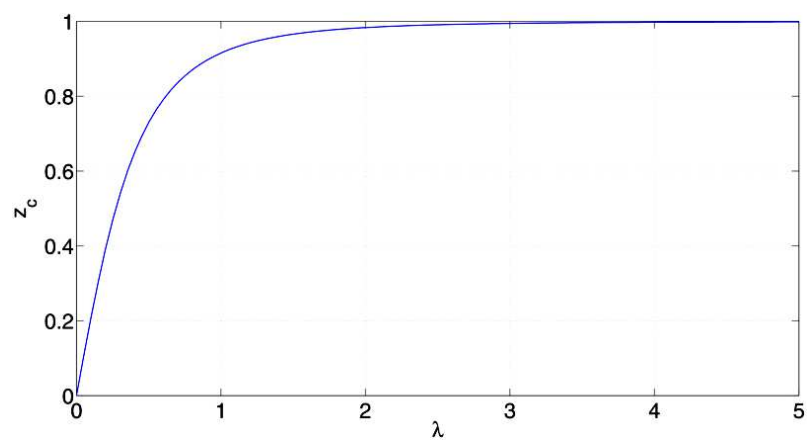

Fig. 4. Plot of $z_{c}(\lambda)$ [Eq. (95)] versus $\lambda$ for $\gamma_{c}=0.4, \kappa=0.8$, and $\gamma=2$

confined in a relatively small frequency interval. We have

$\bar{n}_{c \pm \lambda}=\bar{n}_{c} z_{c}(\lambda)$

where $z_{c}(\lambda)$ is given by

$z_{c}(\lambda)=\frac{2}{\pi}\left[\frac{\kappa}{\kappa-\left(\gamma+\gamma_{c}\right)} \tan ^{-1}\left(\frac{2 \lambda}{\gamma+\gamma_{c}}\right)-\right.$

$\left.-\frac{\left(\gamma+\gamma_{c}\right)}{\kappa-\left(\gamma+\gamma_{c}\right)} \tan ^{-1}\left(\frac{2 \lambda}{\kappa}\right)\right]$. 
Table 1. Values of $\boldsymbol{z}_{a}(\boldsymbol{\lambda})$

and the corresponding local mean photon numbers

\begin{tabular}{|l|l|l|}
\hline$\lambda$ & $z_{a}(\lambda)$ & $\bar{n}_{a \pm \lambda}$ \\
\hline 1.0 & 0.861 & $0.861 \bar{n}_{a}$ \\
2.0 & 0.963 & $0.963 \bar{n}_{a}$ \\
3.0 & 0.985 & $0.985 \bar{n}_{a}$ \\
4.0 & 0.993 & $0.993 \bar{n}_{a}$ \\
5.0 & 0.996 & $0.996 \bar{n}_{a}$ \\
\hline
\end{tabular}

Table 2. Values of $\boldsymbol{z}_{\boldsymbol{b}}(\boldsymbol{\lambda})$

and the corresponding local mean photon numbers

\begin{tabular}{|c|c|c|}
\hline$\lambda$ & $z_{b}(\lambda)$ & $\bar{n}_{b \pm \lambda}$ \\
\hline 1.0 & 0.868 & $0.868 \bar{n}_{b}$ \\
2.0 & 0.965 & $0.965 \bar{n}_{b}$ \\
3.0 & 0.986 & $0.986 \bar{n}_{b}$ \\
4.0 & 0.994 & $0.994 \bar{n}_{b}$ \\
5.0 & 0.997 & $0.997 \bar{n}_{b}$ \\
\hline
\end{tabular}

Table 3. Values of $\boldsymbol{z}_{c}(\boldsymbol{\lambda})$

and the corresponding local mean photon numbers

\begin{tabular}{|l|l|l|}
\hline$\lambda$ & $z_{c}(\lambda)$ & $\bar{n}_{c \pm \lambda}$ \\
\hline 1.0 & 0.917 & $0.917 \bar{n}_{c}$ \\
2.0 & 0.984 & $0.984 \bar{n}_{c}$ \\
3.0 & 0.994 & $0.994 \bar{n}_{c}$ \\
4.0 & 0.998 & $0.998 \bar{n}_{c}$ \\
5.0 & 0.999 & $0.999 \bar{n}_{c}$ \\
\hline
\end{tabular}

We see from Eq. (94) and the plot of $z_{c}(\lambda)$ that $\bar{n}_{c \pm \lambda}$ increases with $\lambda$ until it reaches the maximum value given by Eq. (81).

From Figs. 2, 3, and 4 and Tables 1, 2, and 3, we observe that a large part of the total local mean photon number is confined in a relatively small frequency interval.

In the next subsection, we will study the photonnumber variance of light modes $a, b$, and $c$ in the entire frequency interval, as well as the given frequency interval.

\subsection{Global photon number variance}

Here, we will, determine the global photon number variance. To begin with, the photon-number variance of light mode $a$ at any time $t$ is given by

$\Delta n_{a}^{2}=\left\langle\left(\hat{a}^{\dagger} \hat{a}\right)^{2}\right\rangle-\left(\left\langle\hat{a}^{\dagger} \hat{a}\right\rangle\right)^{2}$.

214
Since $\hat{a}(t)$ is the Gaussian variable, we can rewrite the above expression as [19]

$\left\langle\hat{a}^{\dagger} \hat{a} \hat{a}^{\dagger} \hat{a}\right\rangle=\left\langle\hat{a}^{\dagger} \hat{a}\right\rangle\left\langle\hat{a}^{\dagger} \hat{a}\right\rangle+\left\langle\hat{a}^{\dagger} \hat{a}^{\dagger}\right\rangle\langle\hat{a} \hat{a}\rangle+\left\langle\hat{a}^{\dagger} \hat{a}\right\rangle\left\langle\hat{a} \hat{a}^{\dagger}\right\rangle$.

Now, by substituting Eq. (97) back into (96), we get

$\Delta n_{a}^{2}=\left\langle\hat{a}^{\dagger 2}\right\rangle\left\langle\hat{a}^{2}\right\rangle+\left\langle\hat{a}^{\dagger} \hat{a}\right\rangle\left\langle\hat{a} \hat{a}^{\dagger}\right\rangle$.

Following the same line of reasoning and a procedure like that in Subsection (3.1), one can arrive at

$\left\langle\hat{a} \hat{a}^{\dagger}\right\rangle=\frac{\gamma_{c}}{\kappa}\left\langle\hat{N}_{b}\right\rangle+1$

and

$\left\langle\hat{a}^{2}\right\rangle=\left\langle\hat{a}^{\dagger 2}\right\rangle=0$.

Finally, in view of Eq. (79) along with (99) and (100), the photon-number variance of light mode $a$ at the steady state turns out to be

$\Delta n_{a}^{2}=\frac{\gamma_{c}}{\kappa}\left\langle\hat{N}_{a}\right\rangle\left[\frac{\gamma_{c}}{\kappa}\left\langle\hat{N}_{b}\right\rangle+1\right]$

In view of (65) and (66), the photon number variance of light mode $a$ has, at the steady state, the form

$$
\begin{aligned}
& \Delta n_{a}^{2}=\left[\frac{\gamma_{c}}{\kappa} N\right]^{2}\left[\frac{r_{a}\left(\gamma+\gamma_{c}\right)}{\left(\gamma+\gamma_{c}\right)^{2}+r_{a}\left(\gamma+2 \gamma_{c}\right)}\right] \times \\
& \times\left[\frac{r_{a} \gamma_{c}}{\left(\gamma+\gamma_{c}\right)^{2}+r_{a}\left(\gamma+2 \gamma_{c}\right)}\right]+ \\
& +\left[\frac{\gamma_{c}}{\kappa} N\right]\left[\frac{r_{a}\left(\gamma+\gamma_{c}\right)}{\left(\gamma+\gamma_{c}\right)^{2}+r_{a}\left(\gamma+2 \gamma_{c}\right)}\right] .
\end{aligned}
$$

We note that. in the absence of spontaneous emission, the photon number variance of light mode $a$ reduces to

$\Delta n_{a}^{2}=\bar{n}_{a}^{2}+\bar{n}_{a}$

From (79) and (102), we conclude that the photon statistics of light mode $a$ is super-Poissonian. We also note that light mode $a$ is in a chaotic state.

Similarly, the photon-number variance of light mode $b$, in the entire frequency interval, is found at the steady state to be

$\Delta n_{b}^{2}=\frac{\gamma_{c}}{\kappa}\left\langle\hat{N}_{b}\right\rangle\left[\frac{\gamma_{c}}{\kappa}\left\langle\hat{N}_{c}\right\rangle+1\right]$

ISSN 2071-0194. Ukr. J. Phys. 2021. Vol. 66, No. 3 
In view of Eqs. (80) and (81), Eq. (104) can be rewritten as

$$
\begin{aligned}
& \Delta n_{b}^{2}=\left[\frac{\gamma_{c}}{\kappa} N\right]^{2}\left[\frac{r_{a} \gamma_{c}}{\left(\gamma+\gamma_{c}\right)^{2}+r_{a}\left(\gamma+2 \gamma_{c}\right)}\right] \times \\
& \times\left[\frac{r_{a} \gamma_{c}^{2}}{\left[\gamma+r_{a}\right]\left[\left(\gamma+\gamma_{c}\right)^{2}+r_{a}\left(\gamma+2 \gamma_{c}\right)\right]}\right]+ \\
& +\left[\frac{\gamma_{c}}{\kappa} N\right]\left[\frac{r_{a} \gamma_{c}}{\left(\gamma+\gamma_{c}\right)^{2}+r_{a}\left(\gamma+2 \gamma_{c}\right)}\right] .
\end{aligned}
$$

We note that, for $\gamma=0$ and $r_{a}=\gamma_{c}$, one can easily get

$\Delta n_{b}^{2}=\bar{n}_{b}^{2}+\bar{n}_{b}$,

which is the photon-number variance for chaotic light. To the best of our knowledge, the photon-number variance of light mode $b$ for $\gamma=0$ and $r_{a} \ll \gamma_{c}$ yields

$\Delta n_{b}^{2}=\bar{n}_{b}$.

From (81) and (106), we conclude that the photon statistics of light mode $b$ is super-Poissonian. Relations (81) and (107) imply that that the photon statistics of light mode $b$ is Poissonian.

Similarly, one can easily verify that

$\Delta n_{c}^{2}=\left[\frac{\gamma_{c}}{\kappa}\left(\left\langle\hat{N}_{a}\right\rangle+\left\langle\hat{N}_{b}\right\rangle\right)\right]\left[\frac{\gamma_{c}}{\kappa}\left(\left\langle\hat{N}_{b}\right\rangle+\left\langle\hat{N}_{c}\right\rangle\right)+2\right]+$

$+\left[\frac{\gamma_{c}}{\kappa}\left\langle\hat{m}_{c}^{\dagger}\right\rangle\right]\left[\frac{\gamma_{c}}{\kappa}\left\langle\hat{m}_{c}\right\rangle\right]$.

From the results obtained above, we have observed that the global photon-number variance of light mode $b$ is less than that of light mode $a$, and the photonnumber variance of the two-mode cavity light does not happen to be the sum of the photon-number variances of the separate single-mode cavity light beams.

\subsection{Local photon-number variance}

To determine the photon-number variance of the cavity light, we need to consider the spectrum of photon-number fluctuations of the light mode (see Fig. 5). The spectrum of photon-number fluctuations of light mode $a$ with the central frequency $\omega_{0}$ is expressible as [21]

$S_{a}(\omega)=\frac{1}{\pi} \operatorname{Re} \int_{0}^{\infty} d \tau e^{i\left(\omega-\omega_{0}\right) \tau}\left\langle\hat{n}_{a}(t), \hat{n}_{a}(t+\tau)\right\rangle_{s s}$,

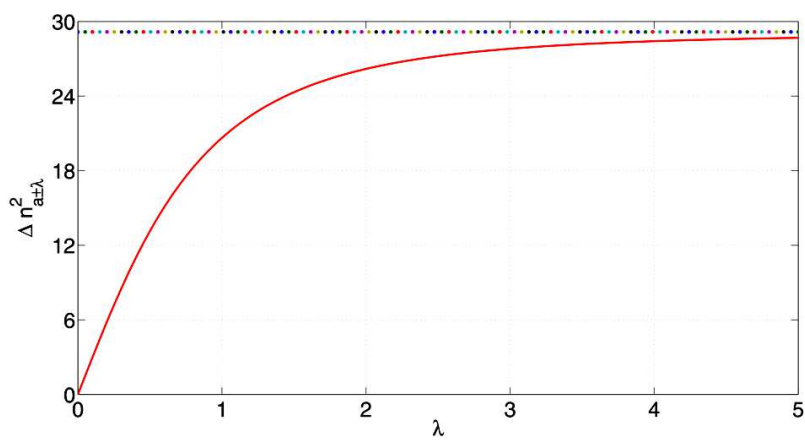

Fig. 5. Plot of the local photon number variance of light mode $a$ [Eq. (119)] versus $\lambda$ for $\gamma_{c}=0.4, \kappa=0.8, r_{a}=2, N=50$, and $\gamma=2$

where

$\hat{n}_{a}(t)=\hat{a}^{\dagger}(t) \hat{a}(t)$

and

$\hat{n}(t+\tau)=\hat{a}^{\dagger}(t+\tau) \hat{a}(t+\tau)$.

Upon integrating both sides of Eq. (109) over $\omega$, we easily find

$\int_{-\infty}^{\infty} S_{a}(\omega) d \omega=\Delta n_{a}^{2}$

where $\Delta n_{a}^{2}$ is the photon-number variance of light mode $a$ at the steady state. We can then assert that $S_{a}(\omega) d \omega$ is the photon-number variance of light mode $a$ in the interval between $\omega$ and $\omega+d \omega$ at the steady state [21].

Next, we need to evaluate the two-time correlation function that appears in Eq. (109). To this end, in view of Eqs. (110) and (111), we readily obtain

$\left\langle\hat{n}_{a}(t), \hat{n}_{a}(t+\tau)\right\rangle=\left\langle\hat{a}^{\dagger}(t) \hat{a}(t+\tau)\right\rangle\left\langle\hat{a}(t) \hat{a}^{\dagger}(t+\tau)\right\rangle+$ $+\langle\hat{a}(t) \hat{a}(t+\tau)\rangle\left\langle\hat{a}^{\dagger}(t) \hat{a}^{\dagger}(t+\tau)\right\rangle$.

It can also be established in a similar manner that

$\left\langle\hat{a}(t) \hat{a}^{\dagger}(t+\tau)\right\rangle=\left\langle\hat{a} \hat{a}^{\dagger}\right\rangle\left[\frac{\kappa}{\kappa-2 \mu} e^{-\mu \tau}-\frac{2 \mu}{\kappa-2 \mu} e^{-\frac{\kappa}{2} \tau}\right]-$

$-\frac{\kappa}{\kappa-2 \mu}\left[e^{-\mu \tau}-e^{-\frac{\kappa}{2} \tau}\right]$

and

$\langle\hat{a}(t) \hat{a}(t+\tau)\rangle=\left\langle\hat{a}^{2}\right\rangle\left[\frac{\kappa}{\kappa-2 \mu} e^{-\mu \tau}-\frac{2 \mu}{\kappa-2 \mu} e^{-\frac{\kappa}{2} \tau}\right]$. 
Here $\mu=\gamma+\gamma_{c}$. Upon substituting Eqs. (85), (114), and (115) into Eq. (113), we get

$$
\begin{aligned}
& \left\langle\hat{n}_{a}(t), \hat{n}_{a}(t+\tau)\right\rangle_{s s}=\Delta n_{a}^{2}\left[\left(\frac{\kappa}{\kappa-2 \mu}\right)^{2} e^{-2 \mu \tau}-\right. \\
& \left.-\frac{4 \kappa \mu}{(\kappa-2 \mu)^{2}} e^{-\left(\frac{\kappa+2 \mu}{2}\right) \tau}+\left(\frac{2 \mu}{\kappa-2 \mu}\right)^{2} e^{-\kappa \tau}\right]-\frac{\bar{n}_{a}}{(\kappa-2 \mu)^{2}} \times \\
& \times\left[\kappa^{2} e^{-2 \mu \tau}-\left(\kappa^{2}+\kappa \mu\right) e^{-\left(\frac{\kappa+2 \mu}{2}\right) \tau}+\kappa \mu e^{-\kappa \tau}\right] .
\end{aligned}
$$

Therefore, in view of Eq. (116), the spectrum of photon-number fluctuations, after performing the integration, takes the form

$$
\begin{aligned}
& S_{a}(\omega)=\left(\frac{\Delta n_{a}}{\kappa-2 \mu}\right)^{2}\left[\frac{2 \kappa^{2} \mu / \pi}{(2 \mu)^{2}+\left(\omega-\omega_{0}\right)^{2}}-\right. \\
& \left.-\frac{4 \mu \kappa(\kappa+2 \mu) / 2 \pi}{\left(\frac{\kappa+2 \mu}{2}\right)^{2}+\left(\omega-\omega_{0}\right)^{2}}+\frac{4 \mu^{2} \kappa / \pi}{\kappa^{2}+\left(\omega-\omega_{0}\right)^{2}}\right]- \\
& -\frac{\bar{n}_{a}}{(\kappa-2 \mu)^{2}}\left[\frac{2 \kappa^{2} \mu / \pi}{(2 \mu)^{2}+\left(\omega-\omega_{0}\right)^{2}}-\right. \\
& \left.-\frac{\left(\kappa^{2}+\mu \kappa\right)(\kappa+2 \mu) / 2 \pi}{\left(\frac{\kappa+2 \mu}{2}\right)^{2}+\left(\omega-\omega_{0}\right)^{2}}+\frac{\mu \kappa^{2} / \pi}{\kappa^{2}+\left(\omega-\omega_{0}\right)^{2}}\right] .
\end{aligned}
$$

Now, we realize that the photon-number variance in the frequency interval between $\omega^{\prime}=-\lambda$ and $\omega^{\prime}=\lambda$ is expressible as

$$
\Delta n_{a \pm \lambda}^{2}=\int_{-\lambda}^{\lambda} S_{a}\left(\omega^{\prime}\right) d \omega^{\prime}
$$

where $\omega^{\prime}=\omega-\omega_{0}$. Therefore, the substitution of Eqs. (117) into (118), after carrying out the integration and making use of (88), gives

$$
\begin{aligned}
& \Delta n_{a \pm \lambda}^{2}=\Delta n_{a}^{2}\left[\frac{2}{\pi}\left(\frac{\kappa}{\kappa-2 \mu}\right)^{2} \tan ^{-1}\left(\frac{\lambda}{2 \mu}\right)-\right. \\
& -\frac{2}{\pi} \frac{4 \kappa \mu}{(\kappa-2 \mu)^{2}} \tan ^{-1}\left(\frac{2 \lambda}{\kappa+2 \mu}\right)+ \\
& \left.+\frac{2}{\pi}\left(\frac{2 \mu}{\kappa-2 \mu}\right)^{2} \tan ^{-1}\left(\frac{\lambda}{\kappa}\right)\right]- \\
& -\frac{\bar{n}_{a}}{(\kappa-2 \mu)^{2}}\left[\frac{2}{\pi} \kappa^{2} \tan ^{-1}\left(\frac{\lambda}{2 \mu}\right)-\right.
\end{aligned}
$$

$\left.-\frac{2}{\pi}\left(\kappa^{2}+\kappa \mu\right) \tan ^{-1}\left(\frac{2 \lambda}{\kappa+2 \mu}\right)+\frac{2}{\pi} \kappa \mu \tan ^{-1}\left(\frac{\lambda}{\kappa}\right)\right]$.

In order to investigate the dependence of the local photon number variance on the frequency of the generated light, the number of the atoms available in the cavity, the stimulated emission decay constant, the spontaneous emission decay constant, the pumping rate, and cavity damping constant, we will plot the local photon number variance versus these parameters. To this end, we only consider the dependence of the local photon number variance on the frequency of the generated light, while the number of the atoms available in the cavity, the stimulated emission decay constant, the spontaneous emission decay constant, the pumping rate, and cavity damping constant are fixed. Based on this, we only plot the photon number variance given by Eq. (119) versus $\lambda$ in which we observe that a large part of the total local mean photon number is confined in a relatively small frequency interval.

\section{Quadrature Squeezing}

In this section, we will study the quadrature variance and the quadrature squeezing of the cavity light modes produced by an electrically driven three-level laser with an open cavity and coupled to a two-mode vacuum reservoir via a single-port mirror. Applying the steady-state solutions of the equations of evolution of the expectation values of the atomic operators and the quantum Langevin equations for the cavity mode operators, we obtain the global quadrature variances of the separate single-mode light beams. In addition, we will determine the global quadrature squeezing of the two-mode cavity light.

\subsection{Quadrature variance}

Here, we will calculate the global quadrature variances of light modes $a$ and $b$ produced by the system under consideration. To begin with, we need to determine the quadrature variance of light mode $a$ in the entire frequency interval. The squeezing properties of light mode $a$ is described by two quadrature operators of the form [14]

$\hat{a}_{+}=\hat{a}^{\dagger}+\hat{a}$

ISSN 2071-0194. Ukr. J. Phys. 2021. Vol. 66, No. 3 
and

$\hat{a}_{-}=i\left(\hat{a}^{\dagger}-\hat{a}\right)$.

These operators are Hermitian and satisfy the commutation relation

$\left[\hat{a}_{-}, \hat{a}_{+}\right]=2 i\left[\frac{\gamma_{c}}{\kappa}\left(\hat{N}_{a}-\hat{N}_{b}\right)-1\right]$.

The operators $\hat{a}_{+}$and $\hat{a}_{-}$represent physical quantities called the plus and minus quadratures, respectively, while $\hat{a}^{\dagger}$ and $\hat{a}$ are the creation and annihilation operators of light mode $a$. The uncertainty relation for the quadrature operators can be expressed as [1]

$\Delta a_{+} \Delta a_{-} \geq\left|\frac{\gamma_{c}}{\kappa}\left(\left\langle\hat{N}_{a}\right\rangle-\left\langle\hat{N}_{b}\right\rangle\right)-1\right|$,

so that, in view of Eqs. (65) and (66), the uncertainty relation for the quadrature operators of cavity light mode $a$ has, at the steady-state, the form

$\Delta \hat{a}_{+} \Delta \hat{a}_{-} \geq\left|\frac{\gamma_{c}}{\kappa}\left(\frac{r_{a} \gamma}{\left(\gamma+\gamma_{c}\right)^{2}+r_{a}\left(\gamma+2 \gamma_{c}\right)}\right) N-1\right|$.

Moreover, we consider the case where the atoms are not bombarded from either the ground or bottom levels to the top level. Thus, by setting $r_{a}=0$, wwe get Eq. (124) in the form

$\Delta a_{+} \Delta a_{-} \geq 1$

Next, we proceed to calculate the quadrature variance of cavity light mode $a$. The quadrature variance of cavity light mode $a$ is defined by

$\Delta \hat{a}_{ \pm}^{2}=\left\langle\hat{a}_{ \pm}^{2}\right\rangle-\left\langle\hat{a}_{ \pm}\right\rangle^{2}$.

With the aid of Eq. (120) and (121), the quadrature variance can be expressed as

$\Delta \hat{a}_{ \pm}^{2}=\left\langle\hat{a} \hat{a}^{\dagger}\right\rangle+\left\langle\hat{a}^{\dagger} \hat{a}\right\rangle \pm\left\langle\hat{a}^{2}\right\rangle \pm$

$\pm\left\langle\hat{a}^{\dagger 2}\right\rangle \mp\langle\hat{a}\rangle^{2} \mp\left\langle\hat{a}^{\dagger}\right\rangle^{2}-2\langle\hat{a}\rangle\left\langle\hat{a}^{\dagger}\right\rangle$.

Now, recalling that the expectation value of $\hat{a}$ is zero and substituting Eqs. (80), (99), and (100) into

ISSN 2071-0194. Ukr. J. Phys. 2021. Vol. 66, No. 3
Eq. (127), the quadrature variance of light mode $a$, at the steady state, takes the form

$\Delta \hat{a}_{ \pm}^{2}=\frac{\gamma_{c}}{\kappa}\left[\frac{r_{a}\left(\gamma+2 \gamma_{c}\right)}{\left(\gamma+\gamma_{c}\right)^{2}+r_{a}\left(\gamma+2 \gamma_{c}\right)}\right] N+1$.

Furthermore, in the absence of spontaneous emission, the quadrature variance can be put in the form

$\Delta \hat{a}_{ \pm}^{2}=\frac{\gamma_{c}}{\kappa}\left[\frac{2 r_{a} \gamma_{c}}{\gamma_{c}^{2}+2 r_{a} \gamma_{c}}\right] N+1$

This quadrature variance can also be expressed as

$\Delta \hat{a}_{ \pm}^{2}=2 \bar{n}_{a}+1$,

which is the quadrature variance for chaotic light in an arbitrary ordering. Moreover, we consider the case where the atoms are not pumped from the ground or bottom levels. Thus, by setting $r_{a}=0$ in either Eq. (128) or (129), we readily get

$\hat{a}_{ \pm \mathrm{vac}}^{2}=1$,

where vac represents the vacuum state, and Eq. (131) is an arbitrary ordered quadrature variance of light mode $a$ in the vacuum state. We note that, for $r_{a}=$ $=0$, the uncertainties in the plus and minus quadratures are equal and satisfy the minimum uncertainty relation.

Similarly, the uncertainty relation for the quadrature operators of cavity mode $b$ has, at the steadystate, the form

$$
\begin{aligned}
& \Delta \hat{b}_{+} \Delta \hat{b}_{-} \geq \mid \frac{\gamma_{c}}{\kappa}\left[\frac{r_{a} \gamma_{c}}{\left(\gamma+\gamma_{c}\right)^{2}+r_{a}\left(\gamma+2 \gamma_{c}\right)}\right] \times \\
& \times\left[1-\frac{\gamma_{c}}{r_{a}+\gamma}\right] N-1 \mid .
\end{aligned}
$$

Moreover, we consider the case where the atoms are not bombarded either from the ground or bottom levels to the top level. Thus, upon setting $r_{a}=0$, Eq. (132) reduces to

$\Delta \hat{b}_{+} \Delta \hat{b}_{-} \geq 1$.

In the absence of spontaneous emission, expression (132) reads reduces to

$\Delta \hat{b}_{+} \Delta \hat{b}_{-} \geq\left|\frac{\gamma_{c}}{\kappa}\left[\frac{r_{a}}{\gamma_{c}+2 r_{a}}\right]\left[1-\frac{\gamma_{c}}{r_{a}}\right] N-1\right|$. 


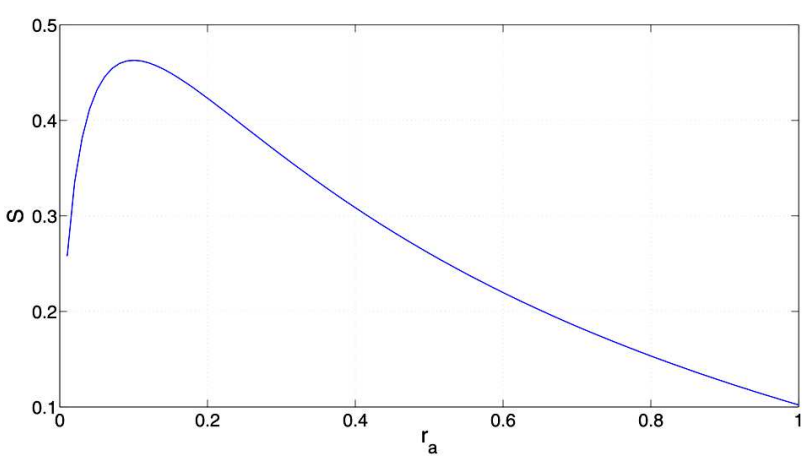

Fig. 6. Plot of the global quadrature squeezing of the twomode cavity light versus $r_{a}$ [Eq. (150)] for $\kappa=0.8, N=50$ $\gamma=0$, and $\gamma_{c}=0.4$

Now, since $\gamma_{c} / r_{a}=\chi$, the above equation takes the form

$\Delta \hat{b}_{+} \Delta \hat{b}_{-} \geq\left|\frac{\gamma_{c}}{\kappa}\left[\frac{1-\chi}{2+\chi}\right] N-1\right|$.

For $\chi \gg 1$ well below the threshold, we immediately observe that the product of uncertainties yields

$\Delta \hat{b}_{+} \Delta \hat{b}_{-} \geq \frac{\gamma_{c}}{\kappa} N+1$.

The quadrature variance of light mode $b$, at steady state, can also take the form

$$
\begin{aligned}
& \Delta \hat{b}_{ \pm}^{2}=\frac{\gamma_{c}}{\kappa}\left[\frac{r_{a} \gamma_{c}}{\left(\gamma+\gamma_{c}\right)^{2}+r_{a}\left(\gamma+2 \gamma_{c}\right)}\right] \times \\
& \times\left[1+\frac{\gamma_{c}}{r_{a}+\gamma}\right] N+1
\end{aligned}
$$

Furthermore, in the case where the spontaneous emission is absent, $\gamma=0$, the quadrature variance can be put in the form

$\Delta \hat{b}_{ \pm}^{2}=\frac{\gamma_{c}}{\kappa}\left[\frac{\chi+1}{\chi+2}\right] N+1$.

We easily see that, for $\chi \gg 1$, the above expression simplifies to

$\Delta \hat{b}_{ \pm}^{2}=\frac{\gamma_{c}}{\kappa} N+1$.

We note that, in the absence of spontaneous emission and well below the threshold, the uncertainties in the plus and minus quadrature are equal and satisfy the minimum uncertainty relation as indicated in Eqs. (136) and (139).

218

\subsection{Quadrature squeezing}

The squeezing properties of light mode $c$ are described by two quadrature operators of the form [14]

$\hat{c}_{+}=\hat{c}^{\dagger}+\hat{c} \quad$ and $\quad \hat{c}_{-}=i\left(\hat{c}^{\dagger}-\hat{c}\right)$.

These operators are Hermitian and satisfy the commutation relation

$\left[\hat{c}_{-}, \hat{c}_{+}\right]=2 i\left[\frac{\gamma_{c}}{\kappa}\left(\hat{N}_{a}-\hat{N}_{c}\right)-2\right]$.

The uncertainty relation for the quadrature operators can be expressed as

$\Delta \hat{c}_{+} \Delta \hat{c}_{-} \geq\left|\frac{\gamma_{c}}{\kappa}\left(\left\langle\hat{N}_{a}\right\rangle-\left\langle\hat{N}_{c}\right\rangle\right)-2\right|$,

so that, in view of Eqs. (65) and (67), the uncertainty relation for the quadrature operators of cavity mode $c$ has, at steady-state, the form

$$
\begin{aligned}
& \Delta \hat{c}_{+} \Delta \hat{c}_{-} \geq \mid \frac{\gamma_{c}}{\kappa}\left[\frac{r_{a}}{\left(\gamma+\gamma_{c}\right)^{2}+r_{a}\left(\gamma+2 \gamma_{c}\right)}\right] \times \\
& \times\left[\frac{\gamma+\gamma_{c}-\gamma_{c}^{2}}{r_{a}+\gamma}\right] N-2 \mid .
\end{aligned}
$$

Moreover, in the absence of spontaneous emission, expression, (143) reduces to

$\Delta \hat{b}_{+} \Delta \hat{b}_{-} \geq\left|\frac{\gamma_{c}}{\kappa}\left[\frac{1-\chi}{2+\chi}\right] N-2\right|$

For $\chi \gg 1$, the product of uncertainties yields

$\Delta \hat{c}_{+} \Delta \hat{c}_{-} \geq \frac{\gamma_{c}}{\kappa} N+2$.

Next, we proceed to calculate the quadrature variance of light mode $c$. The quadrature variance of light mode $c$, with the aid of $\left\langle\hat{m}_{c}\right\rangle=\sqrt{\left\langle\hat{N}_{a}\right\rangle\left\langle\hat{N}_{c}\right\rangle}$, is found, at the steady state, to be

$$
\begin{aligned}
& \Delta c_{ \pm}^{2}=\frac{\gamma_{c}}{\kappa}\left[1+\frac{r_{a} \gamma_{c}}{\left(\gamma+\gamma_{c}\right)^{2}+r_{a}\left(\gamma+2 \gamma_{c}\right)} \times\right. \\
& \left.\times\left(1 \pm 2 \sqrt{\frac{\gamma+\gamma_{c}}{\gamma+r_{a}}}\right)\right] N+2 .
\end{aligned}
$$

Furthermore, in the absence of spontaneous emission, this expression reduces to

$\Delta c_{ \pm}^{2}=\frac{\gamma_{c}}{\kappa}\left[1+\frac{1 \pm 2 \sqrt{\chi}}{2+\chi}\right] N+2$.

ISSN 2071-0194. Ukr. J. Phys. 2021. Vol. 66, No. 3 
We note that, for $\chi \gg 1$, this expression further reduces to

$\Delta c_{ \pm}^{2}=\frac{\gamma_{c}}{\kappa} N+2$.

On the basis of Eqs. (145) and (148), we note that the Heisenberg uncertainties in the two-mode quadrature are equal and satisfy the minimum uncertainty relation [6]. Thus, the three-level laser under consideration generates two-mode coherent light, when it operates under the condition $\gamma=0$ and $\chi \gg 1$.

We immediately notice that the two-mode cavity light is in a squeezed state, and the squeezing occurs in the minus quadrature. To this end, the quadrature squeezing of the two-mode cavity light relative to the quadrature variance of the two-mode coherent state in the entire frequency interval is given by $[3,6]$

$$
S=\frac{\left(\Delta \hat{c}_{-}\right)_{\mathrm{coh}}^{2}-\left(\Delta \hat{c}_{-}\right)^{2}}{\left(\Delta \hat{c}_{-}\right)_{\mathrm{coh}}^{2}}=1-\frac{\left(\Delta \hat{c}_{-}\right)^{2}}{\left(\Delta \hat{c}_{-}\right)_{\mathrm{coh}}^{2}} \text {. }
$$

By substituting the quadrature variance defined by the minus parts of (146) and (148) into (149), the steady-state global quadrature squeezing of the twomode cavity light is found to be

$$
\begin{aligned}
& S=1-\left(\frac { \gamma _ { c } } { \kappa } \left[1+\frac{r_{a} \gamma_{c}}{\left(\gamma+\gamma_{c}\right)^{2}+r_{a}\left(\gamma+2 \gamma_{c}\right)} \times\right.\right. \\
& \left.\left.\times\left(1-2 \sqrt{\frac{\gamma+\gamma_{c}}{\gamma+r_{a}}}\right)\right] N+2\right) /\left(\frac{\gamma_{c}}{\kappa} N+2\right) .
\end{aligned}
$$

The inspection of Fig. 6 indicates that, for certain values of $r_{a}$, the degree of squeezing of the twomode cavity light increases with $r_{a}$. Then the degree of squeezing decreases, as $r_{a}$ increases. For example, it is found from the plot that if $r_{a}=0.101$, the maximum quadrature squeezing of the two-mode cavity light is found to be $46.3 \%$ below the coherent-state level. This global quadrature squeezing is slightly less than that of the global quadrature squeezing discussed in Ref. [12], in which the maximum global quadrature squeezing is $50 \%$ below the coherent state level. This difference is resulted from the arbitrary ordering of the noise operators associated with the vacuum reservoir.

\section{Conclusion}

We have studied the statistical and squeezing properties of the cavity light generated by the threelevel laser. In this optical system, $N$ three-level atoms available in a cavity coupled to a two-mode vacuum reservoir are pumped to the top level from the ground and bottom levels by means of the electron bombardment. We consider the case where the three-level atoms, as well as the cavity light, are interacting with the two-mode vacuum reservoir.

Employing the master equation, we have obtained the quantum Langevin equation for the cavity mode and atomic operators. Applying the steady-state solutions of the equations of evolution of the expectation values of the atomic operators and the quantum Langevin equations, we obtained the global and local photon statistics of the single-mode light beams, as well as the two-mode light beam. In the absence of spontaneous emission, we have noticed that the global mean photon number of light mode $a$ is just equals to the global mean photon number of light mode $b$. On the other hand, in the presence of spontaneous emission, we have noticed that the global mean photon number of light mode $a$ is just greater than the global mean photon number of light mode $b$. Moreover, it is also found that the global mean photon number of the two-mode cavity light is just the sum of the global mean photon numbers of the separate single-mode cavity lights. Furthermore, we have determined the global and local photon-number variance for the single-mode cavity light beams and the two-mode cavity light. From the results, we hav obtained, we have observed that the global photonnumber variance of light mode $a$ is greater than that of light mode $b$, and the photon-number variance of the two-mode cavity light does not happen to be the sum of the photon-number variances of the separate single-mode cavity light beams. In addition, we have also observed that a large part of the local mean photon number and the local photon number variance are confined in a relatively very small frequency interval.

Moreover, we have established the global quadrature squeezing of the two-mode cavity light. Although light modes $a$ and $b$ are separately in a chaotic state, it turns out that the two-mode cavity light is in a squeezed state under certain conditions with a maximum quadrature squeezing of $46 \%$ below the coherent state-level.

1. M.O. Scully, M.S. Zubairy. Quantum Optics (Cambridge Univ. Press, 1997).

2. T. Abebe. The quantum analysis of nondegenerate threelevel laser with spontaneous emission and noiseless vacuum reservoir. Ukr. J. Phys. 63, 969 (2018). 
3. Fesseha Kassahun. Refind Quantum Analysis of Light (CreateSpace Independent Publish. Platform, 2014).

4. Sintayehu Tesfa. Entanglement amplification in a nondegenerate three-level cascade laser. Phys. Rev. A 74, 043816 (2006).

5. C.M. Caves, B.L. Schumaker. New formalism for twophoton quantum optics. I. Quadrature phases and squeezed states. Phys. Rev. A 31, 3068 (1985).

6. M.M. Gessesse. The noise effect of vacuum reservoir on the dynamics of three-level laser pumped by coherent light. Ukr. J. Phys. 65, 385 (2020).

7. M.O. Scully, M.S. Zubairy. Noise free amplification via the two-photon correlated spontaneous emission laser. Opt. Commun. 66, 303 (1988).

8. N.A. Ansari, J. Gea-Banacloche, M.S. Zubairy. Phasesensitive amplification in a three-level atomic system. Phys. Rev. A 41, 5179 (1990).

9. Sintayehu Tesfa. Coherently driven two-level atom coupled to a broadband squeezed vacuum. J. Modern Optics $\mathbf{5 4}$ (12), 1759 (2007).

10. N.A. Ansari. Effect of atomic coherence on the second- and higher-order squeezing in a two-photon three-level cascade atomic system. Phys. Rev. A 48, 4686 (1993).

11. J. Anwar, M.S. Zubairy. Quantum-statistical properties of noise in a phase-sensitive linear amplifier. Phys. Rev. A 49, 481 (1994).

12. Fesseha Kassahun. Three-level laser dynamics with the atoms pumped by electron bombardment. arXiv:1105.1438 v3 [quant-ph], (2012).

13. S. Getahun. Entanglement formulation in the framework of electrically pumped laser cavity. Intern. J. Modern Phys. B 13, 1650024 (2016).

14. G.S. Agarwal. Quantum Optics (Cambridge Univ. Press, 2013).

15. W.H. Louisell. Quantum Statistical Properties of Radiation (Wiley, 1973).

16. Eyob Alebachew, K. Fesseha. Interaction of a two-level atom with squeezed light. Opt. Commun. 271, 154 (2007).

17. Z. Ficek, M.R. Wahiddin. Quantum Optics for Beginners (Stanford Publ., 2014)

18. S.M. Barnett, P.M. Radmore. Methods in Theoretical Quantum Optics (Oxford Univ. Press, 1997).
19. C.W. Gardiner, P. Zoller. Foundation of Quantum Optics (Imperial College Press, 2013).

20. M. Weissbluth. Photon-Atom Interactions (Academic Press, 1989).

21. F. Kassahun. Stimulated emission by two-level atoms pumped to the upper level. Opt. Commun. 284, 1357 (2011).

Received 20.05.20

М. Молла Гесесе

ДИНАМІКА ДВОМОДОВОГО ЛАЗЕРА 3 ЕЛЕКТРИЧНОЮ НАКАЧКОЮ

Досліджено статистичні властивості та стиснення світла у резонаторі трирівневого лазера. У відкритому резонаторі лазера $N$ трирівневих атомів взаємодіють 3 двомодовим вакуумним резервуаром і накачуються на верхній рівень за допомогою електронного бомбардування. Застосовуючи стаціонарні розв'язки рівнянь еволюції для середніх значень атомних операторів і квантові рівняння Ланжевена для операторів мод резонатора, ми отримали загальну і локальну статистики фотонів для світлових променів з однота двомодових резонаторів. Встановлено, що загальне середнє число фотонів і дисперсія числа фотонів, що випромінюються з верхнього рівня, більші, ніж для світла, що випромінюється з проміжного рівня. Світло резонатора, що випромінюється з верхнього і проміжного рівнів, може бути окремо в хаотичному стані при певних умовах. Однак світло з двомодового резонатора при певних умовах може бути в стисненому стані. Ми встановили, що максимальне квадратурне стиснення світла двомодового резонатора буде приблизно на $46 \%$ нижче порівняно із когерентним станом. Наявність шуму вакуумного резервуара збільшує дисперсію числа фотонів і зменшує квадратурне стиснення світла резонатора, але не змінюе середне число фотонів. Ми показали, що локальні значення середнього числа фотонів i дисперсії числа фотонів резонатора наближаються до загальних значень при збільшенні частотного інтервалу.

Ключові слова: константа розпаду стимульованої емісії фотонів, статистика фотонів, спектр потужності, флуктуації кількості фотонів, квадратурне стиснення. 